\title{
Mental-health-related admissions to the acute medical unit during COVID-19
}

\author{
Authors: Ben Grimshaw ${ }^{A}$ and Ella Chaudhuri ${ }^{B}$
}

\begin{abstract}
During COVID-19 there has been increased pressure on mental health services internationally. In this report, we compare admissions to one acute medical unit (AMU) for patients with mental health problems during the COVID-19 pandemic (April, May and June 2020) to the same period of time in 2019. We found an increase in this cohort of patients in 2020 , both as an absolute number and as a proportion of the medical take. We outline some strategies which we have adopted locally to improve care for this patient group.
\end{abstract}

KEYWORDS: acute medicine, mental health, AMU, COVID-19

DOI: 10.7861/clinmed.2020-0635

\section{Introduction}

The global COVID-19 pandemic has increased strain on the mental health of individuals and on the delivery of mental health services to support those with pre-existing mental illness. ${ }^{1,2}$

Studies have also highlighted the effects on the mental health of healthcare workers. ${ }^{3}$ There has been national recognition of these problems, with attempts at mitigation. ${ }^{4}$ However, as the situation has evolved, it has become clear that an increased proportion of our work on the AMU involves managing mental health problems and their physical consequences. Here we quantify the increase in mental-health-related admissions compared to the same period of time in 2019. We also highlight strategies we have adopted locally to improve care in this cohort of patients and promote the safety and wellbeing of our staff.

\section{Methods}

Local Qlik Sense data, conforming to national clinical coding standards, are recorded for all patients admitted to the AMU. Patient-level data from April, May and June 2019 and 2020 were analysed. ICD-10 codes for all patients were reviewed. A search strategy was devised to capture 'mental-health-related admissions' - defined as patients who were referred to, or discussed with, our mental health liaison team in relation to the presenting

Authors: ${ }^{A}$ consultant in acute medicine and stroke, North Bristol NHS Trust, Bristol, UK; ${ }^{B}$ Consultant in acute medicine, North Bristol NHS Trust, Bristol, UK

\begin{tabular}{|c|c|c|c|c|}
\hline Year & Month & $\begin{array}{l}\text { Total } \\
\text { number } \\
\text { of AMU } \\
\text { attendances }\end{array}$ & $\begin{array}{l}\text { Cases } \\
\text { found by } \\
\text { search } \\
\text { strategy }\end{array}$ & $\begin{array}{l}\text { Mental- } \\
\text { health-related } \\
\text { admissions (on } \\
\text { clinical review) }\end{array}$ \\
\hline \multirow[t]{4}{*}{2019} & April & 1,993 & 167 & 22 \\
\hline & May & 1,957 & 172 & 31 \\
\hline & June & 1,910 & 148 & 15 \\
\hline & & & Total & 68 \\
\hline \multirow[t]{4}{*}{2020} & April & 876 & 82 & 27 \\
\hline & May & 900 & 81 & 38 \\
\hline & June & 1,180 & 99 & 41 \\
\hline & & & Total & 106 \\
\hline
\end{tabular}

complaint. Patients who developed psychiatric symptoms as inpatients were not considered in this analysis. The following search terms were applied to each dataset: 'poisoning', 'mental and behavioural disorder', 'anorexia', 'visual', 'hypokalaemia' and 'observation'. These search terms were predicted to best capture deterioration in pre-existing mental health conditions and the physical consequences of mental illness. Cases were drawn from both primary diagnosis and primary diagnosis chapter data entries. Duplicate cases (identified by hospital number) were deleted. Case notes were reviewed for each highlighted care episode to ensure the clinical presentation was related to mental health.

\section{Results}

The number of cases identified at each stage of the search strategy is shown in Table 1. Despite the decreased total number of acute medicine attendances in 2020 compared to 2019, the number of confirmed mental-health-related admissions remained high.

The number of mental-health-related admissions confirmed on clinical review was plotted against date for April, May and June in 2019 and 2020 (Fig 1). This shows the number of mental-healthrelated admissions to the AMU for April and May was similar in both 2019 and 2020, despite a marked decrease in total number of AMU attendances in 2020. In June 2020 the number of admissions related to mental health increased compared to June 2019 (Fig 1). 


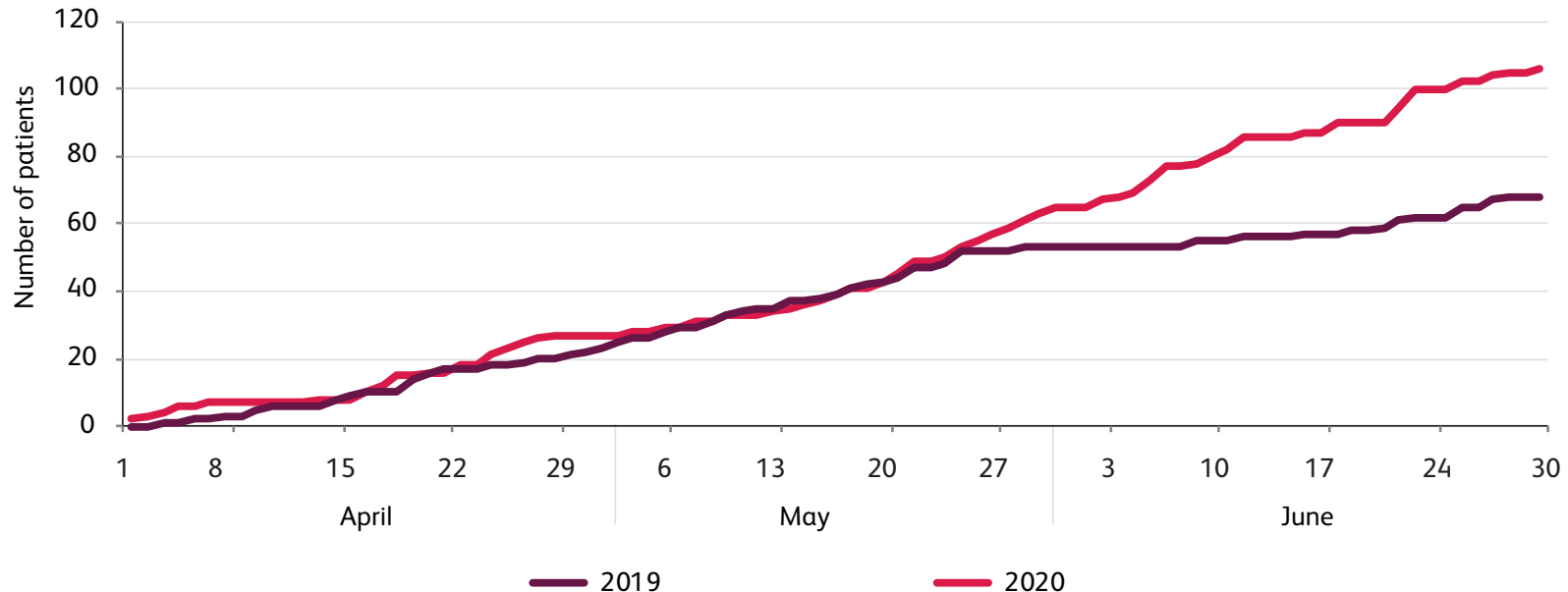

Fig 1. Cumulative Mental-health-related admissions plotted against time (2019 and 2020).

The number of mental-health-related admissions was plotted as a proportion of the total number of admissions to AMU (Fig 2). This shows an increase in these admissions as a proportion of the medical take for 2020 compared to 2019.

The total number of mental-health-related admissions was plotted for April, May and June 2019 and 2020. This shows an increase in the total number of these admissions in 2020 compared with 2019 for each month analysed (Fig 3).

The incidence of mental-health-related admissions over each 3-month study period increased from 0.9 cases per 1,000 person years in 2019 to 1.4 cases per 1,000 person years in 2020 .

The proportion of mental-health-related admissions in each search category was plotted for 2019 and 2020. This shows a broader range of Mental-health-related presentations in 2020 compared to 2019 (Fig 4).

\section{Discussion}

The number of mental-health-related admissions to our AMU has increased in 2020 compared to 2019. There has been an increase in both the absolute number of admissions and admissions as a proportion of the overall medical take. These observations have been made during the COVID-19 pandemic and support the work of other groups which suggest that COVID-19 is a significant stressor. ${ }^{5}$

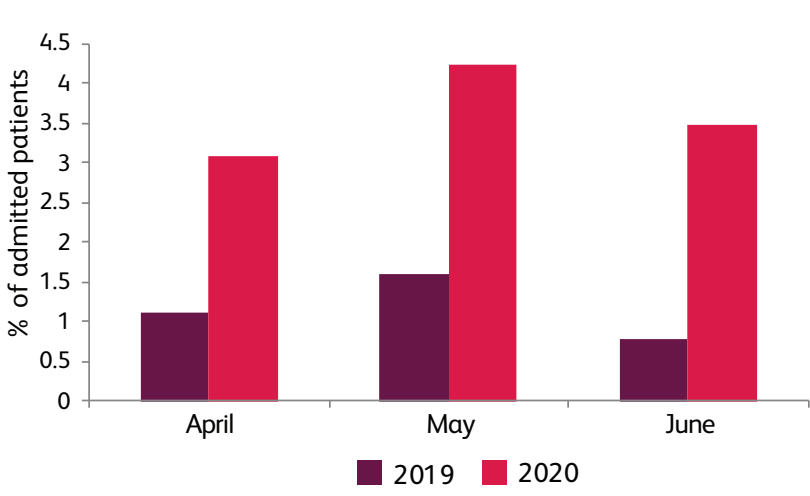

Fig 2. Mental-health-related admissions as a proportion of the medical take for 3 months in 2019 and 2020.

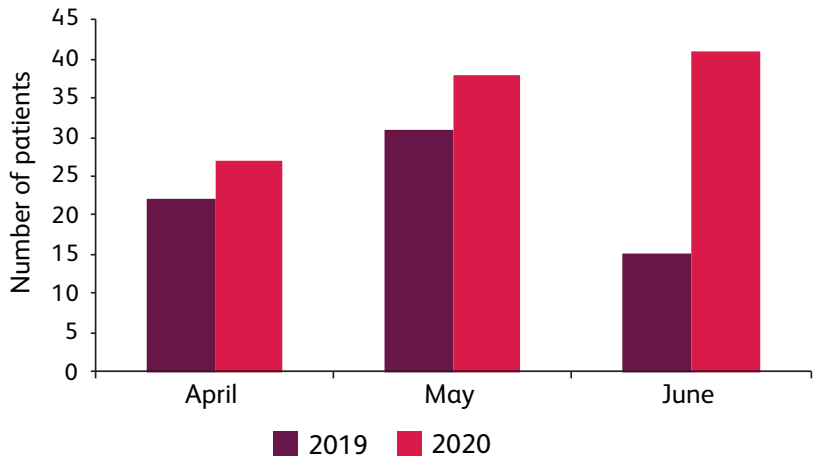

Fig 3. Monthly mental-health-related admissions for 3 months in 2019 and 2020.

A potential limitation of this analysis is that duplicate data entries, based on hospital number, were deleted as part of the search strategy. This was done to ensure cases were not doublecounted (because data was drawn from two different coding data series). However, if a single patient attended more than once, only one admission would be included in the analysis. This could have led to an underestimation of the number of mental health cases recorded above - though the conclusion of an increasing number of cases in 2020 still stands.

Another potential limitation is that our emergency department has reconfigured three observation beds into a minor injuries area during the COVID-19 pandemic. Some patients who require a period of monitoring following deliberate self-poisoning are consequently referred to AMU. This may be confounding a comparison of 2019 and 2020 patient numbers. However, this reconfiguration has been in place since 1 April 2020 and does not explain the sustained rise in patients attending with mental health problems through April, May and June 2020.

The total number of monthly admissions to our AMU has decreased markedly during the COVID-19 pandemic. Despite this, the number of admissions relating to deteriorating mental health continues to rise. Factors driving this increase are beyond the scope of this work, but possibly include interruption to local 


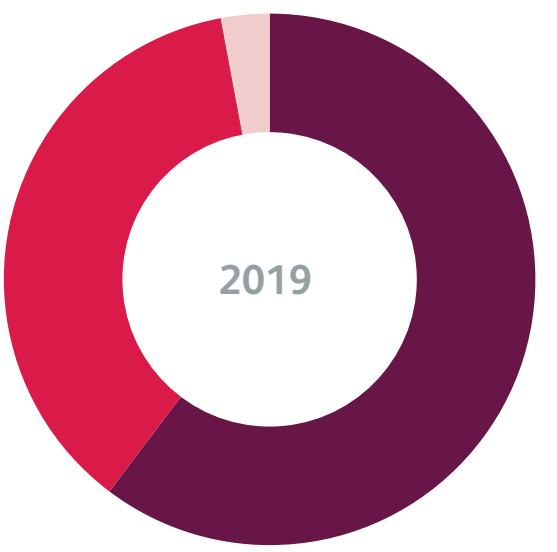

Poisoning
Mental and behabioural disorder

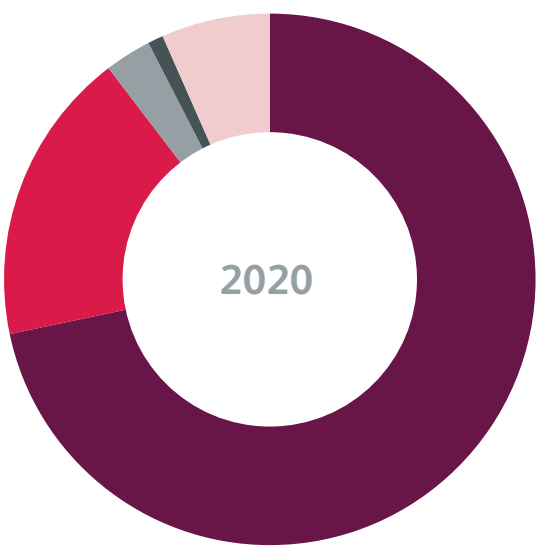

Anorexia
Visual symptoms
Hypokalaemia

Fig 4. Proportion of patients in each mental health coding category for 2019 and 2020.

delivery of mental health support services, telephone consultations replacing face-to-face appointments and support group work taking place via remote access (or being cancelled altogether). Anxiety and 'reactive' mental disorders in the context of COVID-19 may also play a role.

Within AMU, this cohort of patients can present specific challenges. Even a small increase in the number of mental-healthrelated admissions can have large implications. Increased medical and nursing staff time is typically required to manage patients with mental health admissions who are exhibiting escalating behaviour. This circumstance may require verbal de-escalation techniques. In some cases input from hospital security is necessary, along with rapid tranquilisation supervised by medical staff. Similarly, complex capacity and assessments under the Mental Health Act are time consuming, requiring an in-depth exploration of patient comprehension and understanding. There is a risk that patients may harm themselves (or others) while under our care and, when this does occur, more time is required to support staff and analyse the root cause of any episode of harm. There is an effect of stress on staff, with a requirement to debrief after difficult or dangerous situations.

\section{Local measures taken}

In order to address these issues, we have implemented several changes in our local practice.

As of June 2020, we have been using a mental health early warning score (MHEWS) for patients who are at risk of deterioration in their mental state during an AMU admission. This locally developed observation tool aims to identify deteriorating patients and trigger an intervention, thus avoiding crisis point. We deliver ongoing training for nursing staff in the implementation of this tool. We have a designated ligature-free room in an area of high visibility for nursing staff in which potentially harmful furnishings (shower rail, hoist tracking, clothes hooks) have also been removed. We have a local rapid tranquilisation policy, developed in conjunction with our psychiatric liaison team. We also have a mental health link nurse (dual trained as a registered nurse and registered mental health nurse) who has dedicated time to deliver 1:1 training. Sessions can be trainee-led or focused on implementation of our rapid tranquilisation policy.
MAYBO training (a conflict management and physical intervention course) had been implemented for our staff prior to the pandemic. This has now been paused due to social distancing restrictions. However, we are currently exploring options to restart this training with a mix of reduced class numbers and remote access.

We encourage debriefs after significant events relating to mental health. These can be 'hot' debriefs (immediately after the event) or 'cold' debriefs (at a pre-arranged time). Remote meeting methods have driven an increased attendance for cold debriefs as staff no longer need to attend in person to contribute.

Our trust clinical psychology team has started a course of resilience training with band 6 and 7 nurses on the AMU.

\section{Conclusion}

Our local data indicate an increase in the number of patients presenting to $\mathrm{AMU}$ with problems related to mental health during the COVID-19 pandemic. It is possible that this also reflects the experience of other units nationally. It is appropriate, now more than ever, to consider how we can deliver high-quality acute medical care to this vulnerable cohort of patients.

\section{References}

1 Torales J, O'Higgins M, Castaldelli-Maia JM et al. The outbreak of COVID-19 coronavirus and its impact on global mental health. Int J Soc Psychiatry 2020;66:317-20.

2 Ettman C, Abdalla SM, Cohen GH et al. Prevalence of depression symptoms in US adults before and during the COVID-19 pandemic. JAMA Network Open 2020;3:e2019686.

$3 \mathrm{Ng}$ Q, De Deyn MLZQ, Lima DY et al. 2020. The wounded healer: A narrative review of the mental health effects of the COVID-19 pandemic on healthcare workers. Asian J Psychiatr 2020;54:102258.

4 Mental Health UK. Managing your mental health during the coronavirus outbreak. https://mentalhealth-uk.org/help-and-information/ covid-19-and-your-mental-health/ [Accessed 1 July 2020].

5 Fancourt D, Steptoe A. COVID-19 social study. https://www. nuffieldfoundation.org/project/covid-19-social-study.

Address for correspondence: Dr Ben Grimshaw, Southmead Hospital, Southmead Road, Westbury-on-Trym, Bristol BS10 5NB, UK.

Email: ben.grimshaw@nbt.nhs.uk 